\title{
ON LEFT BIPOTENT NEAR-RINGS
}

\author{
by J. L. JAT and S. C. CHOUDHARY \\ (Received 12th September 1977)
}

\section{Introduction}

A near-ring $N$ is defined to be left bipotent if $N a=N a^{2}$ for each $a$ in $N$. Many properties of such near-rings are proved in Section 1, and results of Chandran (4) are generalised. Most of the results are different from, and contrary to, the ring case. Necessary and sufficient conditions have also been obtained under which such near-rings become regular. Section 2 deals with left bipotent near-rings without zero divisors. Some structure theorems for direct sum decompositions and $J(N)=(0)$ are proved and it is shown that for a left bipotent $S$-near-ring, the singular 'set' $S(N)=0$. Necessary examples and counter examples are supplied.

For completeness, the standard definitions are summarised. A near-ring $N$ is a system $(N,+,$.$) such that (N,+)$ is a group (not necessarily abelian), $(N,$.$) is a semigroup, the right$ distributive law holds, i.e. $(x+y) z=x z+y z$ for each $x, y, z$ in $N$; and $x \cdot 0=0$ for every $x$ in $N$. We will take $N$ to be a near-ring containing at least two elements. An element $a$ in $N$ is said to be distributive, if $a(b+c)=a b+a c$ for all $b$ and $c$ in $N ; N$ is called distributively generated (d.g.) (8), if the additive group of $N$ is generated by the multiplicative semigroup of distributive elements of $N$.

An additive group $A$ of $N$ is called a left $N$-subgroup (right $N$-subgroup) if $N A \subseteq A$ $(A N \subseteq A)$ where $N A=\{r a \mid r \in N, a \in A\} . A$ is a left ideal of $N$ if it is a normal left $N$-subgroup with the condition $r_{1}\left(r_{2}+a\right)-r_{1} r_{2} \in A$ for each $a$ in $A, r_{1}, r_{2}$ in $N$. $A$ is an ideal of $N$ if it is left ideal and $A N \subseteq A$.

A proper ideal is called strictly maximal if it is maximal as a left $N$-subgroup. A near-ring $N$ is called irreducible (simple) if it contains only the trivial left $N$-subgroups (ideals) (0) and $N$ itself. $N$ is called a near-field if it contains an identity and each nonzero element has a multiplicative inverse.

1.

Definition 1.1. A near-ring $N$ is said to be left bipotent if $N a=N a^{2}$ for every $a$ in $N$.

Examples 1.2. Let $N_{1}=\{0,1,2,3\}, N_{2}=\{0,1,2,3,4\}$ and $N_{3}=\{0,1,2,3,4,5,6\}$ with additions defined as addition modulo 4 , modulo 5 , and modulo 7 respectively and 
multiplications by the following tables:

\begin{tabular}{l|llll}
$\cdot$ & 0 & 1 & 2 & 3 \\
\hline 0 & 0 & 0 & 0 & 0 \\
1 & 0 & 3 & 0 & 1 \\
2 & 0 & 2 & 0 & 2 \\
3 & 0 & 1 & 0 & 3
\end{tabular}

\begin{tabular}{l|lllll}
$\cdot$ & 0 & 1 & 2 & 3 & 4 \\
\hline 0 & 0 & 0 & 0 & 0 & 0 \\
1 & 0 & 0 & 4 & 1 & 0 \\
2 & 0 & 0 & 3 & 2 & 0 \\
3 & 0 & 0 & 2 & 3 & 0 \\
4 & 0 & 0 & 1 & 4 & 0
\end{tabular}

\begin{tabular}{l|lllllll}
$\cdot$ & 0 & 1 & 2 & 3 & 4 & 5 & 6 \\
\hline 0 & 0 & 0 & 0 & 0 & 0 & 0 & 0 \\
1 & 0 & 1 & 2 & 4 & 4 & 2 & 1 \\
2 & 0 & 2 & 4 & 1 & 1 & 4 & 2 \\
3 & 0 & 3 & 6 & 5 & 5 & 6 & 3 \\
4 & 0 & 4 & 1 & 2 & 2 & 1 & 4 \\
5 & 0 & 5 & 3 & 6 & 6 & 3 & 5 \\
6 & 0 & 6 & 5 & 3 & 3 & 5 & 6
\end{tabular}

Then $N_{1}, N_{2}, N_{3}$ are near-rings (7). It can be seen that they are left bipotent near-rings.

Definition 1.3. A near-ring $N$ is an $S$-near-ring if $a \in N a$ for each $a \in N$.

This condition is clearly satisfied if $N$ contains a left identity, but there exist near-rings (e.g. $N_{3}$ in 1.2) which are $S$-near-rings with no left identity. Also the near rings $N_{1}$ and $N_{2}$ in 1.2 are left bipotent but not $S$-near-rings and in 1.8 we present a near-ring which is an $S$-near-ring but not left bipotent.

Proposition 1.4. A left bipotent S-near-ring contains no non zero nilpotent elements.

Proof. Let $N$ be a left bipotent $S$-near-ring and $b \in N$ be nilpotent, for some $n$, $b^{n}=0$. Then $b \in N b=N b^{2}=\ldots=N b^{n}$ and $b=0$.

The condition that $N$ be an $S$-near-ring is necessary as is seen from the near-rings $N_{1}$ and $N_{2}$ in 1.2.

In the ring case every nilpotent element of a left bipotent ring is in the Jacobson radical (4). But this type of result is not true in near-rings. For example, in the near-ring $\mathrm{N}_{2}$ of $1.2,1$ and 4 are nonzero nilpotent elements, whereas $J_{0}\left(N_{2}\right)=D\left(N_{2}\right)=J_{1}\left(N_{2}\right)=$ $J_{2}\left(N_{2}\right)=(0)$. (Corresponding to the Jacobson radical $J(R)$ in the ring case, we have four radicals $J_{0}(N), D(N), J_{1}(N), J_{2}(N)$ in a near ring $N$. For these see (14)).

We recall that a near-ring $N$ is regular $(1,10)$ if for each $a$ in $N$, there exists $x$ in $N$ such that $a=a x a$.

Theorem 1.5. Let $N$ be an $S$-near ring, then $N$ is regulariff for each $a(\neq 0)$ in $N$, there exists an idempotent $e\left(\right.$ i.e. $\left.e^{2}=e\right)$ such that $\mathrm{Na}=\mathrm{Ne}$.

Proof. If $N$ is a regular near-ring, then for every $a$ in $N$, there exists $x$ in $N$ such that $a=a x a$. Let $x a=e$, then $e$ is clearly an idempotent and $N a=N e$ (for $N a=N a x a \subseteq$ $N x a=N e \subseteq N a$ ). Conversely, let $N$ be an $S$-near-ring satisfying the given condition. For any $d \in N$, there exists an idempotent $b$ such that $d \in N d=N b$. This gives $d=u b$ for some $u$ in $N$. Also $b \in N b=N d$ gives $b=y d$ for some $y$ in $N$. Therefore, $d y d=u b y d=$ $u b^{2}=u b=d$. Hence $N$ is a regular near ring.

Corollary 1.6. (Beidleman (1) and Ligh (10)). Let $N$ be a near-ring with identity. Then $N$ is regular iff for each $a(\neq 0)$ in $N$, there exists an idempotent e such that $N a=N e$. 
Since there exist near-rings which are $S$-near-rings and contain no identity, (e.g. $N_{3}$ in 1.2), Theorem 1.5 generalises Theorem 2 of (1) and Theorem 4.2 of (10).

In general, left bipotence neither implies nor is implied by the regularity of the near-rings. The near-ring $N_{1}$ of 1.2 is left bipotent but not regular. We now present a near-ring which is regular but not left bipotent.

Example 1.7. Let $R$ be the ring of all $n \times n$ matrices $(n \geqslant 2)$ over a division ring. It can be seen that it is regular but not strongly regular (see Szász (15), $R$ is said to be strongly regular if for each $a \in R, a=y a^{2}$ for some $y$ in $R$ ). Take $N=R \times M$, where $M$ is an $R$-module. Define addition + and multiplication $*$ on $N$ as follows:

$$
\begin{aligned}
& \left(a_{1}, m_{1}\right)+\left(a_{2}, m_{2}\right)=\left(a_{1} a_{2}, m_{1}+m_{2}\right) ; \\
& \left(a_{1}, m_{1}\right) *\left(a_{2}, m_{2}\right)=\left(a_{1} a_{2}, a_{1} m_{2}+m_{1}\right) .
\end{aligned}
$$

Then $(N,+, *)$ is a near-ring with identity $(1,0)$ (see Clay (6)). We assert that this is regular but not left bipotent. Let $(a, m) \in N$. Since $R$ is regular, there exists $b$ in $R$ such that $a=a b a$. Then clearly $(a, m) *(b,-b m) *(a, m)=(a b a, m)=(a, m)$ shows that $N$ is regular. Also, since $R$ is not strongly regular, for some $x$ in $R, x \neq y x^{2}$ for any $y$ in $R$. Taking this $x \in R$ and any $m$ in $M$, then clearly $(x, m) \neq\left(y, m^{\prime}\right) *(x, m)^{2}$ for any $y \in R$ (as $x \neq y x^{2}$ ) and any $m^{\prime}$ in $M$. This shows that $N$ is not left bipotent (since $N$ contains an identity).

Now we give the conditions under which left bipotency implies regularity and vice versa.

Definition 1.8. A near-ring $N$ is said to be strictly duo (duo), if each left $N$-subgroup (left ideal) of $N$ is also a right $N$-subgroup (right ideal).

Clearly each strictly duo near-ring is duo. The converse is true in rings, but not in near-rings. The near-ring

\begin{tabular}{c|cccc}
+ & 0 & $a$ & $b$ & $c$ \\
\hline 0 & 0 & $a$ & $b$ & $c$ \\
$a$ & $a$ & 0 & $c$ & $b$ \\
$b$ & $b$ & $c$ & 0 & $a$ \\
$c$ & $c$ & $b$ & $a$ & 0
\end{tabular}

\begin{tabular}{l|llll}
$\cdot$ & $\mathbf{0}$ & $\mathbf{a}$ & $\mathbf{b}$ & $\mathrm{c}$ \\
\hline $\mathbf{0}$ & $\mathbf{0}$ & $\mathbf{0}$ & $\mathbf{0}$ & $\mathbf{0}$ \\
$\mathbf{a}$ & $\mathbf{0}$ & $\mathrm{a}$ & $\mathrm{b}$ & $\mathrm{c}$ \\
$\mathbf{b}$ & $\mathbf{0}$ & $\mathrm{b}$ & $\mathbf{0}$ & $\mathbf{0}$ \\
$\mathrm{c}$ & $\mathbf{0}$ & $\mathrm{c}$ & $\mathrm{b}$ & $\mathrm{c}$
\end{tabular}

is duo but not strictly duo, since the left $N$-subgroup $\{0, c\}$ is not a right $N$-subgroup. Since this near-ring contains the identity $a$, it is an $S$-near-ring. Moreover it is not left bipotent, as $N b \neq N b^{2}$.

Theorem 1.9. A regular near-ring $N$ is left bipotent if either of the following conditions is satisfied:

(i) $N$ is a strictly duo near-ring,

(ii) Each idempotent in $N$ is central.

Proof. (i) Let $N$ be a regular near ring and let $a \in N$. Then there exists $x$ in $N$ such 
that $a=a x a$. This gives $a=(a x) a x a=(a x)^{2} a$. Since $N$ is strictly duo and $N a$ is a left $N$-subgroup of $N$ we have $N a N \subseteq N a$. Therefore, $(a x) a \cdot x=b a$ for some $b$ in $N$, which with $a=(a x)^{2} a$ gives $a=b a^{2}$. This implies $N a=N a^{2}$ and so $N$ is left bipotent.

(ii) $N$ is regular, so far $a$ in $N$, there exists $x$ in $N$ such that $a=a x a$. Clearly $x a$ is an idempotent, and $a=a x a=x a \cdot a$ (since idempotents are central). Hence $N a=N a^{2}$ and $N$ is left bipotent.

Theorem 1.10. A left bipotent near-ring $N$ is regular iff $N$ is an $S$-near-ring.

Proof. Clearly every regular near-ring is an $S$-near-ring. Conversely, let $N$ be a left bipotent $S$-near-ring. Then for each $a$ in $N a \in N a=N a^{2}=N a^{3}=N a^{4}$ and so $a^{2}=z a^{4}$ for some $z$ in $N$. This gives $\left(a^{2}-a^{2} z a^{2}\right) a^{2}=0$ and $\left(a^{2}-a^{2} z a^{2}\right) a^{2} z a^{2}=0$. Also by $1.4, N$ contains no nonzero nilpotent elements. This implies $a^{2}\left(a^{2}-a^{2} z a^{2}\right)=0$ and $a^{2} z a^{2}\left(a^{2}-\right.$ $\left.a^{2} z a^{2}\right)=0$ and therefore, $\left(a^{2}-a^{2} z a^{2}\right)^{2}=0$. Again applying 1.4, we get $a^{2}=a^{2} z a^{2}$. Then clearly $z a^{2}=e$ is an idempotent and $N a=N a^{2}=N a^{2} z a^{2} \subseteq N z a^{2}=N e \subseteq N a^{2}=N a$. Hence, by Theorem $1.5, N$ is regular.

For any subset $A$ of a near-ring $N$, we define $\sqrt{A}=\left\{x \in N \mid x^{n} \in A\right.$ for some $\left.n\right\}$.

Theorem 1.11. If $N$ is a left bipotent $S$-near-ring, then $A=\sqrt{A}$ for every left $N$-subgroup $A$ of $N$.

Proof. Clearly $A \subseteq \sqrt{A}$. Now let $a \in \sqrt{A}$, then $a^{n} \in A$ for some $n$. Also we have $N a=N a^{2}=-\cdot=N a^{n}$ in a left bipotent near-ring. Since $N$ is an $S$-near-ring, $a \in N a=N a^{n}$. This gives $a=b a^{n}$ for some $b$ in $N$. Thus $a \in A$, (since $a^{n} \in A$ and $A$ is a left $N$-subgroup of $N$ ). Hence $\sqrt{A} \subseteq A$.

The condition that $N$ be an $S$-near-ring in this theorem is necessary, since in the near-ring $N_{2}$ of $1.2(0) \neq \sqrt{(0)}$. We have seen earlier that an $S$-near-ring need not be left bipotent, however we do have:

Theorem 1.12. An S-near-ring is left bipotent iff $A=\sqrt{A}$ for every left $N$-subgroup A of $N$.

Proof. In view of Theorem 1.11 we have to prove that if $N$ is an $S$-near-ring with the condition $A=\sqrt{A}$ for every left $N$-subgroup $A$ of $N$ then $N$ is left bipotent. For $a \in N$, $a^{3} \in N a^{2}$ and $a \in \sqrt{N a^{2}}=N a^{2}$. Then $N a \subseteq N a^{2} \subseteq N a$ and $N$ is left bipotent.

2.

Theorem 2.1. A left bipotent near ring is an S-near ring iff it has no nonzero nilpotent elements.

Proof. A left bipotent $S$-near-ring has no nonzero nilpotent elements by 1.4 . Conversely let $N$ be left bipotent with no nonzero nilpotent elements. If $a, b, c \in N$ with $a b c=0$ then $a c b=0$ (as $a b c=0 \Rightarrow b a b c=0 \Rightarrow c b a b=0 \Rightarrow a c b a b=0 \Rightarrow b a c b a=$ $0 \Rightarrow$ bacbac $=0 \Rightarrow(a c b)^{2}=0 \Rightarrow a c b=0$ ). Now for any $x$ in $N, N x=N x^{2}$ so $x^{2}=y x^{2}$ for some $y$ in $N$. Then $(x-y x)(x-y x)=x(x-y x)-y x(x-y x)$. Since $(x-y x) x=0$ we get 
$x(x-y x)=0$. Also, $y(x-y x) x=0$ so $y x(x-y x)=0$. Hence $(x-y x)^{2}=0$ and $x=y x$ as required.

The near-rings $N_{1}$ and $N_{2}$ in 1.2 show that a left bipotent near-ring with nilpotent elements need not be an $S$-near-ring.

This theorem, with Theorem 1.10, immediately gives:

Corollary 2.2. A left bipotent near-ring is regular iff it has no nonzero nilpotent elements.

Proposition 2.3. A left bipotent near-ringwith no (proper) zero divisors is irreducible.

Proof. Let $A$ be a nonzero left $N$-subgroup of a left bipotent near-ring $N$. Take any nonzero element $a$ in $A$, then $N a=N a^{2}$. If $r \in N$ then $r a=s a^{2}$ for some $s$ in $N$. Therefore $(r-s a) a=0$ and $r=s a$ (as $N$ is without proper divisors of zero). Clearly $r=s a \in A$ and so $A=N$. Thus $N$ is irreducible.

Theorem 2.4. Let $N$ be a left bipotent near-ring with no zero divisors. If $N$ has a nonzero distributive element, then $N$ is a near-field.

Proof. By Corollary 2.2, $N$ is regular (as no zero divisors implies no nilpotent elements). Let $d$ be a nonzero distributive element in $N$, then there exists $x$ in $N$ such that $d=d x d$. Clearly $x d$ (=e say) is an idempotent. If $r$ is any element in $N$, then $(d=d x d) r=0$ or $(d-d e) r=0$ and so $d(r-e r)=0$ (since $d$ is a distributive element). This gives $r=e r$, i.e. $e$ is a left identity in $N$. If $a \in N$ with $a \neq 0$ then $N a=N a^{2}$. Therefore, $e a=y a^{2}$ for some $y$ in $N$. This gives $(e-y a) a=0$ and so $e=y a$, i.e. $y$ is a left inverse of $a$. Hence $N$ is a near-field.

In the above theorem, the existence of a nonzero distributive element is essential, as the near-ring $N_{3}$ in 1.2 is left bipotent without nonzero divisors of zero, but it is not anearfield because the element 3 does not have an inverse.

Corollary 2.5. Let $N$ be a left bipotent distributively generated (d.g.) near-ring with no zero divisors, then $N$ is a division ring.

Proof. From Theorem $2.4 ; N$ is a near-field and so $(N,+)$ is abelian, (see (8)). Moreover, a d.g. near-ring with $(N,+)$ abelian is a ring (12). Therefore, $N$ is a division ring.

Proposition 2.6. A left identity of a left bipotent near-ring is also a right identity.

Proof. Since $N$ contains a left identity (say $e$ ), it is an $S$-near-ring. If $r \in N$, then $r=e r$. This gives $(r-r e) r=0$ and $(r-r e) r e=0$. Then $\{r(r-r e)\}^{2}=r(r-r e) r(r-r e)=$ 0 , and therefore, by $1.4, r(r-r e)=0$. Similarly $r e(r-r e)=0$. These give $(r-r e)^{2}=$ $r(r-r e)-r e(r-r e)=0$ and hence $r=r e$ (by 1.4), i.e. $e$ is also a right identity.

Each of the near-rings $N_{1}, N_{2}, N_{3}$ in 1.2 shows that a right identity of a left bipotent near-ring need not be a left identity. 
For the next structure theorem we need the following result of Heatherly (9). We state it (as a lemma) for (right) near-rings.

Lemma 2.7. If $N$ is a simple near-ring without nilpotent elements and satisfies the d.c.c. on left $N$-subgroups, then (a) every nonzero idempotent of $N$ is a right identity and $N$ has at least one such idempotent; (b) $N$ is regular; $(c)$ if $N$ has a nonzero distributive element, then $N$ is a near-field; (d) if $N$ is d.g., then $N$ is a division ring.

Theorem 2.8. Let $N$ be a left bipotent S-near-ring satisfying the d.c.c. on its left N-subgroups, then

(I) $N$ is a direct sum of regular near-rings with right identities.

(II) If $N$ has a nonzero distributive element, then $N$ is a direct sum of near-fields.

(III) If $N$ is d.g., then $N$ is a direct sum of division rings.

Proof. Since $N$ is a left bipotent $S$-near-ring, it contains no nonzero nilpotent elements (by 1.4) and so no nonzero nilpotent left $N$-subgroups. Since $N$ also satisfies the d.c.c. on its left $N$-subgroups, by Blackett's (3) decomposition theorem, $N$ is a direct sum of ideals $N=N_{1} \oplus N_{2} \oplus \ldots \oplus N_{k}$, where each $N_{i}$, as a near-ring, is simple with the d.c.c. on its left $N_{i}$-subgroups. Clearly each $N_{i}$ contains no nonzero nilpotent elements. This means each $N_{i}$ satisfies the conditions of Lemma 2.7 and therefore each $N_{i}$ is regular with a right identity. This proves $(I)$.

If $N$ has a nonzero distributive element $d$, then $d=d_{1}+d_{2}+\ldots+d_{k}$, where $d_{i} \in N_{i}$ $(1 \leqslant i \leqslant k)$. Then it is easy to see that for each $i(1 \leqslant i \leqslant k)$, the element $d_{i}$ is distributive in the corresponding near-ring $N_{i}$ in the above direct sum decomposition of $N$. Therefore, by Lemma 2.7 (c), each $N_{i}$ is a near-field and so $N$ is a direct sum of near-fields. This proves (II).

If $N$ is a distributively generated (d.g.) near-ring then so is the each direct summand $N_{i}$ in the direct sum decomposition of $N$. Therefore, by Lemma 2.7(d), each $N_{i}$ is a division ring. This proves (III).

Theorem 2.9. If $N$ is a left bipotent near-ring with a left identity and satisfying the d.c.c. on its left $N$-subgroups, then $J(N)=(0)$, where $(J(N)$ is the radical of $N$ (see (2), (5)).

Proof. Since $N$ has a left identity, it is an $S$-near-ring and so it contains no nonzero nilpotent left $N$-subgroups (by 1.4). Also $N$ satisfies the d.c.c. on its left $N$-subgroups and so $N$ is semi-simple in the sense of Blackett (3). The left identity of a left bipotent near-ring is also a right identity (by 2.6). Now apply Theorem 3.1 of (5), which states that for a near-ring $N$ with right identity, semisimplicity in the sense of Blackett is equivalent to the condition that $N$ satisfies the d.c.c. on left ideals and $J(N)=(0)$.

In this theorem a 'left identity' is necessary, as the near-ring $N_{1}$ in 1.2 is left bipotent and finite; but $J(N)=\{0,2\}$.

Definition 2.10. (13) An ideal $P(\neq N)$ is called strictly prime (prime) if for any two left $N$-subgroups (ideals) $A$ and $B$ of $N$ such that $A B \subseteq P$, then $A \subseteq P$ or $B \subseteq P$. 
Accordingly a near-ring is said to be strictly prime (prime), if the zero ideal is a strictly prime (prime) ideal.

Proposition 2.11. A strictly prime left bipotent $S$-near-ring is irreducible.

Proof. In view of Proposition 2.3, it is sufficient to show that $N$ has no nonzero divisors of zero. Let $a b=0$ for $a, b$ in $N$. Then for any $x$ in $N,(b x a)^{2}=0$, which gives $b x a=0$ (by Proposition 1.4). So $N b N a=(0)$. Since $N$ is strictly prime and $N b, N a$ are left $N$-subgroups we have that $N b=(0)$ or $N a=(0)$. Hence, since $N$ is an $S$-near-ring either $b=0$ or $a=0$.

Theorem 2.12. Let $A$ be an ideal of the near-ring $N$. If $N$ is a left bipotent or an $S$-near-ring so also is $N / A$.

The proof is straight forward.

Theorem 2.13. Every strictly prime ideal of a left bipotent S-near-ring is strictly maximal.

Proof. Let $P$ be a strictly prime ideal of a left bipotent $S$-near-ring $N$. Then $N / P$ is also a strictly prime left bipotent $S$-near-ring (by 2.12 ) and hence $N / P$ is irreducible (by 2.11) which gives that $P$ is strictly maximal.

Definition 2.14. (11). A left ideal $B$ of a near-ring $N$ is called strictly essential (essential), if $B \cap K \neq(0)$, for every nonzero left $N$-subgroup (left ideal) $K$ of $N$.

Definition 2.15. (11). An element $x \in N$ is said to be singular, if there exists a nonzero strictly essential left ideal $A$ in $N$ such that $A x=(0)$.

Let $S(N)$ denote the set of all singular elements of $N$ and $l(x)=\{r \in N \mid r x=0\}$. It can be seen that $l(x)$ is a left ideal of $N$.

Lemma 2.16. Let $N$ be a near-ring. Then

$$
S(N)=\{x \in N \mid l(x)
$$

is a nonzero strictly essential left ideal in $N$ \}.

Proof. Let $x \in S(N)$. Then there exists a nonzero strictly essential left ideal $A$ in $N$ such that $A x=(0)$. Therefore, $A \subseteq l(x)$ and so $l(x)$ is a nonzero strictly essential left ideal in $N$. Thus $S(N) \subseteq\{x \in N \mid l(x)$ is a nonzero strictly essential left ideal in $N\}$. The inclusion the other way is direct since $l(x) x=(0)$.

Chandran (4) has shown that for a left bipotent ring $R, S(R)=J(R)$, the Jacobson radical. But this need not be true in near-rings. For example, for the left bipotent near-ring $N_{2}$ in 1.2, all the radicals corresponding to the Jacobson radical are zero, whereas $S(N)=\{0,1,4\}$. This also shows that, contrary to the ring theory case, $S(N)$ need not be an ideal. 
We use the following result of Maxson (11);

Lemma 2.17. For a near-ring $N, N S(N) \subseteq S(N)$.

Theorem 2.18. Let $N$ be a left bipotent near-ring, then

(i) every nilpotent element of $N$ is in $S(N)$,

(ii) if $N$ has no nonzero nilpotent elements, then $S(N)=0$.

Proof. (i) Let $x$ be a nonzero nilpotent element of a left bipotent near-ring $N$. Then $x^{n}=0$ for some $n$, and so $N x=N x^{2}=\ldots=N x^{n}=(0)$. This gives $l(x)=N$. Thus, $l(x)$ is a nonzero strictly essential left ideal in $N$. Therefore, by Lemma 2.16, $x \in S(N)$.

(ii) Let $b$ be a nonzero element in $S(N)$, then $N b=N b^{2}=N b^{3} N b^{4}$, and so there exists $z$ in $N$ such that $b^{2}=z b^{4}$. This gives $\left(b^{2}-b^{2} z b^{2}\right) b^{2}=0$ and $\left(b^{2}-b^{2} z b^{2}\right) b^{2} z b^{2}=0$. Since $N$ contains no nonzero nilpotent elements, we get $b^{2}\left(b^{2}-b^{2} z b^{2}\right)=0$ and $b^{2} z b^{2}\left(b^{2}-b^{2} z b^{2}\right)=0$. This implies $\left(b^{2}=b^{2} z b^{2}\right)^{2}=0$ and hence $b^{2}=b^{2} z b^{2}$. Also $z b^{2}$ is an idempotent (say, $e$ ) and $e=z b^{2}=z b \cdot b \in S(N)$ (by Lemma 2.17). Therefore, by Lemma $2.16, l(e)$ must be a nonzero strictly essential left ideal in $N$. But for the left $N$-subgroup $N e$, we have $l(e) \cap N e=(0)$. This is a contradiction, as $e \in S(N)$. Hence $b=0$ and so $S(N)=0$.

This theorem, with Proposition 1.4, gives the following.

Corollary 2.19. For a left bipotent $S$-near-ring $N, S(N)=0$.

Acknowledgement. The authors wish to express their gratitude to the referee for valuable comments that have improved the presentation of the above results. In particular, Theorem 2.1 and its corollary are due to the referee.

\section{REFERENCES}

(1) J. C. Beidleman, A note on regular near rings, J. Indian Math. Soc. 33 (1969), 207-210.

(2) J. C. BeidlemaN, A radical for near ring modules, Mich. Math. J. 12 (1965), 377-383. 772-785.

(3) D. W. Blacketr, Simple and semisimple near rings, Proc. Amer. Math. Soc. 4 (1953),

(4) V. R. Chandran, On right bipotent rings, Abstract in Notices Amer. Math. Soc. Nov. (1970).

(5) S. C. Choudh ARY and K. Tewari, On strictly semisimple near rings, Abh. Math. Sem. Univ. Hamburg 40 (1974), 256-264.

(6) J. R. CLAY, Imbedding an arbitrary ring in a nontrivial near ring, Amer. Math. Monthly 74 (1967), 406-407.

(7) J. R. Clay, The near rings on groups of low order, Math. Z. 104 (1968), 364-371.

(8) A. FröHlich, Distributively generated near rings (I ideal Theory), Proc. London Math. Soc. 8 (1958), 76-94.

(9) H. E. Heatherly, Near rings without nilpotent elements, Pub. Math. Debrecen 20 (1973), 201-205. 
(10) S. LigH, On regular near rings, Math. Japonicae Ser (1) 15 (1970), 7-13.

(11) C. J. MAxson, On near rings and near ring modules (Ph.D. Dissertation, State Univ. of New York at Buffalo, New York, 1967).

(12) B. H. NeumanN, On the commutativity of addition, J. London Math. Soc. 15 (1940), 203-208.

(13) A. OsWALD, Near rings in which every $N$-subgroup is principal, Proc. London Math. Soc. 28 (1974), 68-88.

(14) D. RAMAKOtAiAH, Radicals for near rings, Math. Z. 97 (1967), 45-56.

(15) F. SzÁsz, A class of regular rings, Monatsh. Math. 75 (1971), 168-172.

Department of Mathematics

SBSH, UNIVERSITY OF UDAIPUR

UDAIPUR-313001, INDIA 